International Journal of Pure and Applied Mathematics

Volume 99 No. 4 2015, 471-476

ISSN: 1311-8080 (printed version); ISSN: 1314-3395 (on-line version)

url: http://www.ijpam.eu

doi: http://dx.doi.org/10.12732/ijpam.v99i4.7

ijpam.eu

\title{
RESULTS OF GENERALIZED LOCAL COHOMOLOGY WITH RESPECT TO A PAIR OF IDEALS
}

\author{
Fatemeh Dehghani-Zadeh \\ Department of Mathematics \\ Islamic Azad University \\ Yazd Branch, Yazd, IRAN
}

\begin{abstract}
Let $(I, J)$ be a pair of ideals of a commutative Noetherian local ring $R$, and $M$ a finitely generated module. Let $t$ be a positive integer. We prove that $(i)$ if $H_{I, J}^{i}(M)$ is minimax for all $i<t$, then $H_{I, J}^{i}(M)$ is $(I, J)$-cofinite for all $i<t$ and $\operatorname{Hom}\left(R / I, H_{I, J}^{t}(M)\right)$ is finitely generated; (ii) if $\mathfrak{a} \in \widetilde{W}(I, J)$ and $H_{I, J}^{i}(M)$ is minimax for all $i<t$, then $\operatorname{Ext}_{R}^{i}(R / \mathfrak{a}, T)$ is minimax for all $i<t$. We also prove that if $\operatorname{Supp} H_{I, J}^{i}(M)=\{\mathfrak{m}\}$ for all $i<t$, then $H_{I, J}^{i}(M)$ is Artinian and $(I, J)$-cofinite for all $i<t$.
\end{abstract}

AMS Subject Classification: 13D45, 13E05, 14B15

Key Words: local cohomology modules, artinian modules, cofinite modules

\section{Introduction}

Throughout this paper, $R$ is denoted a commutative Noetherian ring, $I$, and $J$ are denoted two ideals of $R$, and $M$ is a finitely generated $R$-module. For notations and terminologies that is not given in this paper, the reader is referred to $[1]$.

As a generalization of the ordinary local cohomology modules, Takahashi, Yoshino and Yoshizawa [7] introduced the local cohomology modules with respect to a pair of ideals $(I, J)$. To be more precise, let $W(I, J)=\{\mathfrak{p} \in \operatorname{Spec}(R) \mid$

Received: October 22, 2014

(c) 2015 Academic Publications, Ltd. url: www.acadpubl.eu 
$I^{n} \subseteq \mathfrak{p}+J$ for some positive integer $\left.n\right\}$. Then for an $R$-module $M$, the $(I, J)$ torsion submodule $\Gamma_{I, J}(M)$ of $M$, which consists of all elements $x$ of $M$ with $\operatorname{Supp} R x \subseteq W(I, J)$, is considered. It is known, $\Gamma_{I, J}(\quad)$ is a left exact additive functor from the category of all $R$-modules and $R$-homomorphism to itself. For all integer $i$, the $\mathrm{i}$-th local cohomology functor $H_{I, J}^{i}$ with respect to $(I, J)$ is defined to be the $i$-th right derived functor of $\Gamma_{I, J}()$. The $i$-th local cohomology module of $M$ with respect to $(I, J)$ is denoted by $H_{I, J}^{i}(M)$. When $J=0$, then $H_{I, J}^{i}$ coincides with the usual local cohomology functor $H_{I}^{i}$ with the support in the closed subset $V(I)$.

There are many questions about ordinary local cohomology modules. In particular, Hunke [5] proposed the following question: for an integer $i$, when is $H_{I}^{i}(M)$ Artinian? In [3] Grothendieck conjectured that for any finite $R$ module $M, \operatorname{Hom}_{R}\left(R / I, H_{I}^{i}(M)\right)$ is finite for all $i$. Hartshorne [4] later refined this conjecture, and proposed the following one:

Let $M$ be a finite $R$-module, and let $I$ be an ideal of $R$. Then

$$
\operatorname{Ext}_{R}^{j}\left(R / I, H_{I}^{i}(M)\right)
$$

is finite, for every $i \geq 0$ and $j \geq 0$. The purpose of this paper is to investigate a similar question as above for this generalized version of local cohomology. Obviously, these results are true in the category of all graded $R$-modules and homogeneous $R$-homomorphisms.

\section{The Results}

Definition 1. An $R$-module $T$ is called $(I, J)$-cofinite if $\operatorname{Supp} T \subseteq W(I, J)$ and $\operatorname{Ext}_{R}^{i}(R / I, T)$ is a finite $R$-modules, for every $i \geq 0$.

Lemma 2. Let $T$ be an arbitrary $R$-module. Then the following statements hold:

(i) Suppose that $T$ is a module with support in $V(I)$. Then $T$ is $(I, J)$-cofinite if and only if $T$ is $I$-cofinite.

(ii) $T$ is $(I, J)$-cofinite Artinian ( minimax) if and only if $T$ is $I$-cofinite Artinian (minimax).

(iii) Let $T$ be Artinian. If $\operatorname{Hom}(R / I, T)$ is finite, then $T$ is $(I, J)$-cofinite.

(iv) Let SuppT $\subseteq V(I)$. If there is an element $x \in I$, such that $\left(0:_{T} x\right)$ is Artinian and $(I, J)$-cofinite, then $T$ is Artinian and $(I, J)$-cofinite. 
Proof. (i) and (ii) are clear because $V(\mathfrak{m}) \subseteq V(I) \subseteq W(I, J)$.

(iii) Since, in view of the hypothesis, $\operatorname{Hom}(R / I, T)$ has finite length and SuppT $\subseteq V(\mathfrak{m}) \subseteq V(I)$. By using [6, Proposition 4.1], we can get $\operatorname{Ext}_{R}^{i}(R / I, T)$ is finitely generated for all $i \geq 0$ thus the proof is completed.

(iv) In view of (ii), $\left(0:_{T} x\right)$ is Artinian and $I$-cofinite. Therefore, according to [6, Proposition 4.1] $T$ is Artinian and $I$-cofinite; and hence the result follows.

Therefore, in view of lemma (2, ii) and [6, Corollary 4.4], the class of $(I, J)$ cofinite Artinian modules is closed under taking submodules, quotients and extensions.

Definition 3. An $R$-module $T$ is said to be minimax module, if there is a finitely generated submodule $T_{1}$ of $T$ such that the quotient module $T / T_{1}$ is Artinian. The class of minimax modules includes all finite and all Artinian modules.

Theorem 4. Let $T$ be an arbitrary $R$-module and $t$ a positive integer and let $\mathfrak{a} \in \widetilde{W}(I, J)$, where $\widetilde{W}(I, J)$ denote the set of ideals $\mathfrak{a}$ of $R$ such that $I^{n} \subseteq \mathfrak{a}+J$ for some integer $n$. If $H_{I, J}^{i}(T)$ is Artinian (minimax) for all $0 \leq i<t$, then $\operatorname{Ext}_{R}^{i}(R / \mathfrak{a}, T)$ is Artinian (minimax)for all $0 \leq i<t$.

Proof. We prove by induction on $t$. The case where $t=1$ yields $\Gamma_{I, J}(T)$ is Artinian (minimax). In view of proof $\left[7\right.$,Theorem 3.2], $\Gamma_{\mathfrak{a}}(T) \subseteq \Gamma_{I, J}(T)$. Thus $\Gamma_{\mathfrak{a}}(T)$ is Artinian (minimax). In particular, $\operatorname{Hom}(R / \mathfrak{a}, T)$ is Artinian (minimax). Assume that $t \geq 2$ and the Theorem holds true for $t-1$. It follows from [7, Corollary 1.13] that $H_{I, J}^{i}(T) \cong H_{I, J}^{i}\left(T / \Gamma_{I, J}(T)\right)$ for all $i>0$. Also, $T / \Gamma_{I, J}(T)$ is an $(I, J)$-torsion-free $R$-module. Hence we can (and do) assume that $M$ is an $(I, J)$-torsion-free $R$-module. Thus $\Gamma_{I, J}(T)=0$ implies that $\Gamma_{\mathfrak{a}}(T)=0$. Then there exists $x \in \mathfrak{a}$ such that $x$ is an $T$-sequence. Now, we may consider the exact sequence $\quad 0 \longrightarrow T \stackrel{x}{\longrightarrow} T \longrightarrow T / x T \longrightarrow 0 \quad$ to obtain the exact sequences

$$
\begin{array}{r}
H_{I, J}^{i}(T) \stackrel{x}{\longrightarrow} H_{I, J}^{i}(T) \longrightarrow H_{I, J}^{i}(T / x T) \quad \text { and } \\
\operatorname{Ext}_{R}^{i}(R / \mathfrak{a}, T) \stackrel{x}{\longrightarrow} \operatorname{Ext}_{R}^{i}(R / \mathfrak{a}, T) \longrightarrow \operatorname{Ext}_{R}^{i}(R / \mathfrak{a}, T / x T) .
\end{array}
$$

Now, the above exact sequences are used in conjunction with the inductive hypothesis to see that the $R$ - modules $H_{I, J}^{i}(T / x T)$ and $\operatorname{Ext}_{R}^{i}(R / \mathfrak{a}, T)$ and $\operatorname{Ext}_{R}^{i}(R / \mathfrak{a}, T / x T)$ are Artinian (minimax) for all $i<t-1$. It is enough to 
show that $\operatorname{Ext}_{R}^{t-1}(R / \mathfrak{a}, T)$ is Artinian (minimax). Finally, note that $x \in \mathfrak{a}$, and $(*)$ exact sequence yields that the sequence

$$
\operatorname{Ext}_{R}^{t-2}(R / \mathfrak{a}, T) \longrightarrow \operatorname{Ext}_{R}^{t-2}(R / \mathfrak{a}, T / x T) \longrightarrow \operatorname{Ext}_{R}^{t-1}(R / \mathfrak{a}, T) \longrightarrow 0,
$$

is exact consequently $\operatorname{Ext}_{R}^{t-1}(R / \mathfrak{a}, T)$ is Artinian (minimax), as required.

Lemma 5. Let $T$ is a minimax $R$-module with Supp in $W(I, J)$. Then the following hold:

(i) If $\operatorname{Hom}(R / I, T)$ is finitely generated, then $T$ is $(I, J)$-cofinite.

(ii) If there is an element $x \in I$, such that $\left(0:_{T} x\right)$ is minimax and $(I, J)$ cofinite, then $T$ is minimax and $(I, J)$-cofinite.

Proof. (i) Let $T_{1}$ be a finitely generated submodule of $T$, such that $T_{2}=$ $T / T_{1}$ is Artinian and suppose that $\operatorname{Hom}\left(\frac{R}{T}, T\right)$ is finitely generated. The exactness of

$$
0 \longrightarrow \operatorname{Hom}\left(R / I, T_{1}\right) \longrightarrow \operatorname{Hom}(R / I, T) \longrightarrow \operatorname{Hom}\left(R / I, T_{2}\right) \longrightarrow \operatorname{Ext}_{R}^{1}\left(R / I, T_{1}\right),
$$

implies that $\operatorname{Hom}\left(R / I, T_{2}\right)$ is finitely generated. Hence we get from Lemma (2, iii) and [2, Lemma 2.1] that $T_{2}$ is Artinian and $(I, J)$-cofinite, therefore $T$ is also $(I, J)$-cofinite.

(ii) If $(o: T x)$ minimax and $(I, J)$-cofinite, then $\operatorname{Hom}\left(R / I,\left(0::_{T} x\right) \cong\right.$ $\operatorname{Hom}(R / I, T)$ is finitely generated. It is clear by (i).

Theorem 6. Let $t$ be a non-negative integer, such that $H_{I, J}^{i}(M)$ is $(I, J)$ cofinite minimax for all $i<t$. Then, the $R$-module $\operatorname{Hom}\left(R / I, H_{I, J}^{i}(M)\right)$ is finitely generated for all $i \leq t$.

Proof. Since $H_{I, J}^{i}(M)$ is $(I, J)$-cofinite, $\operatorname{Hom}\left(R / I, H_{I, J}^{i}(M)\right)$ is finitely generated for all $i<t$. So it is enough to prove that $\operatorname{Hom}\left(R / I, H_{I, J}^{t}(M)\right)$ is finitely generated. We prove by induction on $t \geq 0$. If $t=0$ then the result is clear. Assume that $t>0$ and the result holds true for $t-1$. It follows from [7, Corollary 1.13] that $H_{I, J}^{i}(M) \cong H_{I, J}^{i}\left(M / \Gamma_{I, J}(M)\right)$ for all $i>0$. Also $M / \Gamma_{I, J}(M)$ is $(I, J)$-torsion-free $R$-module. Since $\Gamma_{I}(M) \subseteq \Gamma_{I, J}(M)$. We can assume that $M$ is an $I$-torsion-free $R$-module. Then there exists an element $x \in I$ which is $M$-regular. The exact sequence

$$
0 \longrightarrow M \stackrel{x}{\longrightarrow} M \longrightarrow M / x M \longrightarrow o
$$


induces a long exact sequence

$$
H_{I, J}^{i-1}(M) \longrightarrow H_{I, J}^{i-1}(M / x M) \longrightarrow H_{I, J}^{i}(M) \stackrel{x}{\longrightarrow} H_{I, J}^{i}(M) \longrightarrow H_{I, J}^{i}(M / x M) .
$$

Also, by the hypothesis, $H_{I, J}^{i}(M / x M)$ is $(I, J)$-cofinite minimax for all $i<$ $t-1$, so that, by the inductive hypothesis, $\operatorname{Hom}\left(R / I, H_{I, J}^{t-1}(M / x M)\right)$ is finitely generated. On the other hand, the exact sequence

$$
0 \longrightarrow H_{I, J}^{t-1}(M) / x H_{I, J}^{t-1}(M) \longrightarrow H_{I, J}^{t-1}(M / x M) \longrightarrow\left(0:_{H_{I, J}^{t}(M)} x\right) \longrightarrow 0,
$$

induces the exact sequence

$$
\begin{aligned}
0 \longrightarrow & \operatorname{Hom}\left(R / I, H_{I, J}^{t-1}(M) / x H_{I, J}^{t-1}(M)\right) \longrightarrow \operatorname{Hom}\left(R / I, H_{I, J}^{t-1}(M / x M)\right) \\
& \longrightarrow \operatorname{Hom}\left(R / I,\left(0:_{H_{I, J}^{t}(M)} x\right)\right) \longrightarrow \operatorname{Ext}^{1}\left(R / I, H_{I, J}^{t-1}(M) / x H_{I, J}^{t-1}(M)\right)
\end{aligned}
$$

Since $\operatorname{Ext}_{R}^{1}\left(R / I, H_{I, J}^{t-1}(M) / x H_{I, J}^{t-1}(M)\right)$ is finitely generated, it follows from the above exact sequence that $\operatorname{Hom}\left(R / I,\left(0:_{H_{I, J}^{t}(M)} x\right)\right)$ is finitely generated $R$-module. Now, as $x \in I$ we have $\operatorname{Hom}\left(R / I, H_{I, J}^{t}(M)\right)$ is finitely generated, as required.

Lemma 7. Let $t$ be a non-negative integer, such that $H_{I, J}^{i}(M)$ is minimax for all $i<t$. Then $H_{I, J}^{i}(M)$ is $(I, J)$-cofinite for all $i<t$ and $\operatorname{Hom}\left(R / I, H_{I, J}^{t}(M)\right)$ is finitely generated.

Proof. By Theorem (6), we need only to prove that $H_{I, J}^{i}(M)$ is $(I, J)$ cofinite for all $i<t$. We proceed by induction on $i$. It is clear that $H_{I, J}^{0}(M)$ is $(I, J)$-cofinite. Assume that $i>0$ and the result holds true for smaller values than $i$. Thus we obtain that $H_{I, J}^{i}(M)$ is $(I, J)$-cofinite minimax for all $j \leq i-1$ by the inductive hypothesis. It follows by Theorem (6) that $\operatorname{Hom}\left(R / I, H_{I, J}^{i}(M)\right)$ is finitely generated. Therefore by Lemma $(5), H_{I, J}^{i}(M)$ is $(I, J)$-cofinite minimax. Now the assertion follows from Theorem (6).

Corollary 8. Let $t$ be a non-negative integer such that $\operatorname{SuppH}_{I, J}^{i}(M)$ $=\{\mathfrak{m}\}$ for $i<t$. Then $H_{I, J}^{i}(M)$ is Artinian and $(I, J)$-cofinite for $i<t$. Moreover $\operatorname{Hom}\left(R / I, H_{I, J}^{t}(M)\right)$ is finitely generated.

Proof. We now prove the Lemma by induction on $t$. It $t=1$ then it is clear that $\Gamma_{I, J}(M)$ is Artinian and $(I, J)$-cofinite. Assume that $t>1$ and the result holds true for $t-1$. By the inductive hypothesis, the $R$-module $H_{I, J}^{i}(M)$ 
is Artinian and $(I, J)$-cofinite for all $i<t-1$. Therefore, by Theorem (6) $\operatorname{Hom}\left(R / I, H_{I, J}^{t-1}(M)\right)$ is finitely generated $R$-module. Since $\operatorname{Supp} H_{I, J}^{t-1}(M)=$ $\{\mathfrak{m}\}$, it follows that it yields from Lemma $\left(2\right.$, iv) that, $H_{I, J}^{t-1}(M)$ is Artinian and $(I, J)$-cofinite. Now assertion follows from Theorem (6).

\section{Acknowledgments}

The author would like to thank the referees for their careful reading.

\section{References}

[1] M. P. Brodmann and R. Y Sharp, Local cohomology: An Algebraic introduction with geometric applications, Cambridge Univ. Press 1998.

[2] F. Dehghani-Zadeh, On the finiteness properties of generalized local cohomology modules, International Electronic Journal of Algebra, 10 (2011), 113-122.

[3] A. Grothendieck, Cohomological local des faisceaur coherents et théoremes de Lefschetz locaux et globaus (SGA2), North-Holland, Amsterdam, 1968.

[4] R. Hartshorne,' Affine duality and cofiniteness ', Invent. Math, (1970), 145-164.

[5] C. Hunkek, Problems on local cohomology, in: Free resolutions in commutative algebra and algebraic geometry, Research Notes In Math, ed, by Eisenbud. and Huneke C., Jones and Bartlett, Boston, 1992, 93-108.

[6] L. Melkersson, Modules cofinite with respect to an ideal, J. Algebra, 285 (2005), 649-668.

[7] R. Takahashi and Y. Yoshino and T. Yoshinawa, Local cohomology based on a nonclosed support defined by a pair of ideals, J. Pure Apple. Algebra, 213 (2009), 582-600. 